\title{
ANSWER TO QUIZ ON PAGE 92.
}

Correct answer is:

C) Sinus rhythm with blocked atrial extrasystole, followed by several escape atrial beats.

Blocked atrial extrasystole can be clearly observed in leads V1, V3 and in inferior leads II, III, aVF as a sharpening of the T wave.

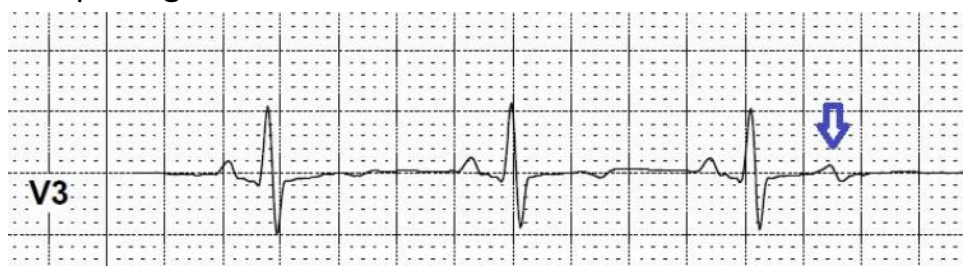

P waves of $4^{\text {th }}$ and $5^{\text {th }}$ PQRST complexes are slightly different than previous $\mathrm{P}$ waves. So they can be considered as right atrial escape beats.

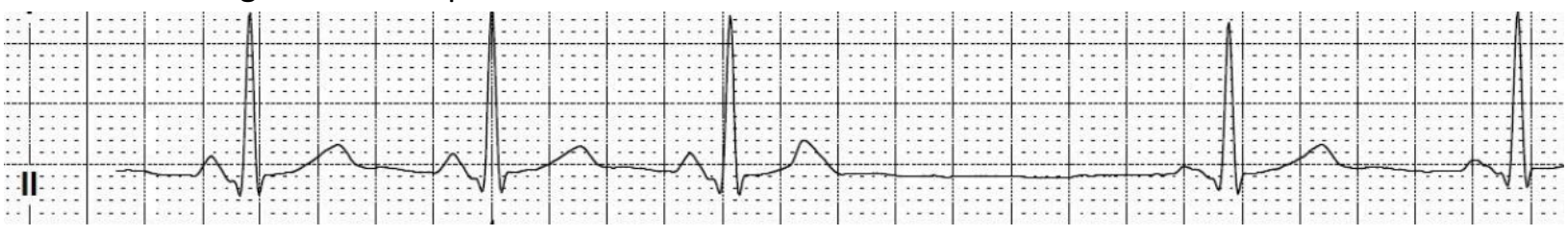

Figure 1. Fragments of multichannel Holter monitoring.

Mykhaylo Sorokivskyy, Lviv Regional Cardiology Center, Danylo Halytsky Lviv National Medical University, Lviv, Ukraine

Peer-review: Internal; Conflict of Interest: None to Declare; Authorship: M.S. Acknowledgement: No funding or material support was received for preparation of the quiz (quiz - page 92)

Address for Correspondence: Mykhaylo Sorokivskyy, Lviv Regional Cardiology Center, Danylo Halytsky Lviv National Medical University, Lviv, Ukraine Email: sorokivskyy@gmail.com Received 10.12.2017 Accepted 10.12.2017 doi: 10.24969/hvt.2017.33

Copyright $\odot 2017$ Heart Vessels and Transplantation 